\title{
MULTIPLE FUNCTIONS LONG TRACE PROFILER (LTP-MF) FOR NATIONAL SYNCHROTRON RADIATION LABORATORY OF CHINA*
}

\author{
Shinan Qian ${ }^{*}$, Qiuping Wang ${ }^{* *}$, Yilin Hong ${ }^{* *}$, and Peter Takacs ${ }^{*}$ \\ *Brookhaven National Laboratory \\ Upton, NY 11973-5000 \\ *** National Synchrotron Radiation Laboratory \\ University of Science and Technology of China \\ Hefei, China
}

July, 2005

*This manuscript has been authored by Brookhaven Science Associates, LLC under Contract No. DE-AC02-98CH10886 with the U.S. Department of Energy. The United States Government retains, and the publisher, by accepting the article for publication, acknowledges, a world-wide license to publish or reproduce the published form of this manuscript, or allow others to do so, for the United States Government purposes. 


\title{
Multiple functions Long Trace Profiler (LTP-MF) for National Synchrotron Radiation Laboratory of China
}

\author{
Shinan Qian", Qiuping Wang ${ }^{* *}$, Yilin Hong**, Peter Takacs* \\ *Brookhaven National Laboratory, Upton, NY 11973, USA \\ ** National Synchrotron Radiation Laboratory \\ University of Science and Technology of China, Hefei, P.R. China
}

\begin{abstract}
The Long Trace Profiler (LTP) is a useful optical metrology instrument for measuring the figure and slope error of cylindrical aspheres commonly used as synchrotron radiation (SR) optics. It is used extensively at a number of synchrotron radiation laboratories around the world. In order to improve SR beam line quality and resolution, the National Synchrotron Radiation Laboratory (NSRL) of China is developing a versatile LTP that can be used to measure both SR optics and more conventional "normal" optical surfaces. The optical metrology laboratories at Brookhaven National Laboratory (BNL) and NSRL are collaborating in developing a multiple functions LTP (LTP-MF). Characteristics of the LTP-MF are: a very compact and lightweight optical head, a large angular test range ( $\pm 16 \mathrm{mrad}$ ) and high accuracy. The LTP-MF can be used in various configurations: as a laboratory-based LTP, an in-situ LTP or penta-prism LTP, as an angle monitor, a portable LTP, and a small radius of curvature test instrument. The schematic design of the compact optical head and a new compact slide are introduced. Analysis of different measurements modes and systematic error correction methods are introduced.
\end{abstract}

\section{INTRODUCTION}

The LTP is a very important optical instrument for measuring synchrotron radiation optics. It is being used in many synchrotron radiation (SR) laboratories around the world $[1,2]$. In order to improve SR beam line quality and resolution for experiments, the National Synchrotron Radiation Laboratory of China at the University of Science and Technology of China (USTC), recognized the need to have an LTP on site. In the Phase I and Phase II construction periods of NSRL, it was very difficult to know whether the delivered optics complied with specifications due to the lack of appropriate metrology instrumentation. Some optical components had to be sent overseas for measurements. For example, during the Phase II period a bending mirror was tested at ESRF for setting the correct surface shape. A

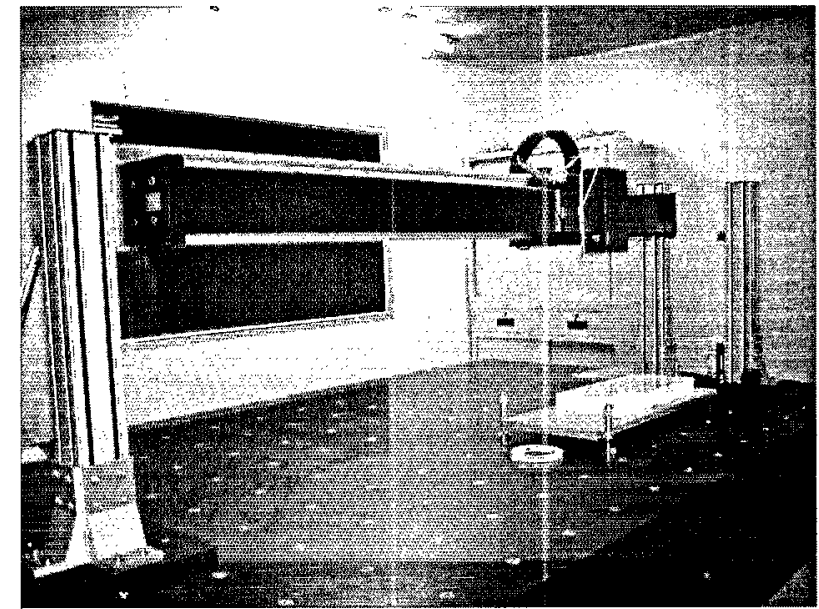

Fig.1: Phase I of LTP-MF: laboratory-based LTP at NSRF in China collaboration was established in June 2004 between the optical metrology laboratories of Brookhaven National Laboratory and NSRL to develop an advanced multifunctions LTP (LTP-MF) for NSRL. At the same time BNL will build LTP-MF for research on improving accuracy, development and calibration.

The characteristics of the LTP-MF are: a very compact and lightweight optical head, an extended angular test range $( \pm 16 \mathrm{mrad})$ and high-accuracy. The multiple functions LTP can be configured as a laboratory-based LTP, a penta-prism LTP [3], an in-situ LTP [4,5], an angle monitor [6], a portable LTP [7] and as a small radius of curvature test instrument. In addition to measuring SR optics, NSRL will also be able to test a range of conventional optical surfaces for USTC and for China. 
The LTP-MF development consists two phases. The goal of phase I is to produce a regular laboratory-based LTP for SR optics measurements. BNL is responsible for the overall system and optical head design; NSRL is responsible for fabricating the LTP optical head according to the BNL design. Ocean Optics, Inc., has provided the control software and a low cost translation stage system to ensure that phase I could be completed quickly. In May 2005 phase I of LTPMF project was finished (Fig.1). The purpose of phase II is to update the laboratory-based LTP to an advanced LTP-MF system, which entails developing new software in order to run the different functions of the LTP-MF, calibrating over a larger angle, systematic error reduction, making a second optical head and a compact slide. According to project, by design, most of the multiple functions characteristics have been included in the existing phase I optical head.

\section{BRIEF DESCRIPTION OF MULTIPLE FUNCTIONS LTP (LTP-MF)}

The multiple functions of the LTP-MF are achieved by several new design characteristics:

1) It has a very compact optical head. Its volume is about $1 / 4$ of that of the LTP II and LTP V, and it follows that its weight is very light. Because of its small size, it can be easily transported for different applications.

2) It has a larger test range of $\pm 16 \mathrm{mrad}$ compared to the range of $\pm 5 \mathrm{mrad}$ of the LTP II and $\pm 10 \mathrm{mrad}$ of the LTP V.

3) It has high-accuracy. The LTP-MF accuracy is expected to be higher because it does not use an inclined reference beam, but does incorporate an extra systematic error correction method and a sine bar calibration.

"Multiple functions" means that the LTP-MF can be used as:

1) A laboratory-based LTP for routine testing in the laboratory [1,2]

2) A penta-prism LTP [3]. It can be setup on a tabletop for precise plano or near-plano surface testing

3) An In-situ LTP $[4,5]$. The compact optical head combined with a specially-designed compact slide is good for in-situ SR heat-load distortion testing and on-beam-line mechanical stability testing of various components.

4) A small angle monitor [6]. It functions like an autocollimator but with the advantage of a strong and small size probe beam for convenient alignment and accurate testing.

5) A portable LTP (PTLTP) [7]. The LTP-MF optical head is much smaller than previous versions. Its size is $200 \mathrm{~mm} \times 112 \mathrm{~mm} \times 60 \mathrm{~mm}$, which is $1 / 2.8$ volume of the previous PTLTP. So now we can package the LTP-MF optical head, compact slide, and laptop computer in only one laptop bag. It is very easy to take anywhere in the world for comparison testing.

6) A conventional optical surface test instrument due to its high accuracy and larger test range. The radius of curvature of optical components used in ordinary optical systems is usually smaller than that of SR optics. With a test range of $\pm 16 \mathrm{mrad}$ with the LTP-MF, we can test a radius of curvature of 1 meter in a $32 \mathrm{~mm}$ aperture without stitching.

\section{SETUP OF THE LTP-MF}

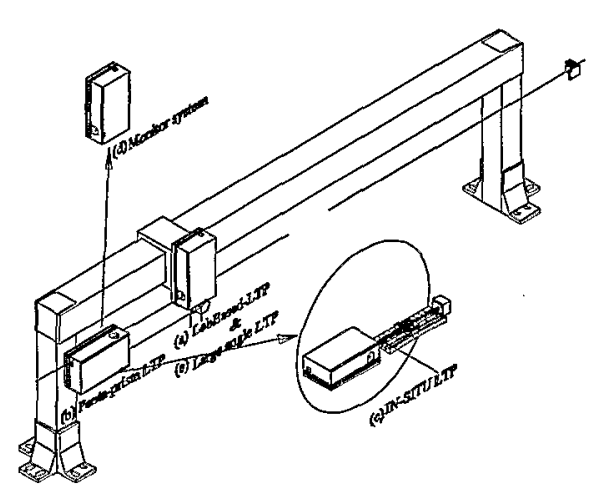

Fig.2: The Multiple Functions LTP (LTP-MF) schematic, showing the various possible configurations.
For maximum performance and for maximum convenience in applications, the LTP-MF consists of the following major sub-systems: two optical heads, one air-bearing slide with a travel of 1.5 meters, one compact slide with a travel of $120 \mathrm{~mm}$, one penta-prism scan unit, and control and analysis software.

A schematic diagram of the various LTP-MF system configurations is shown on Fig.2. One LTPMF optical head is fixed to the carriage of the 1.5 meter long air bearing (Fig. 2 (a)). This configuration is used for laboratory-based LTP measurements. The optical head is fixed to the scanning carriage to avoid detaching and realigning the optical head if there was only one optical head. 
On the right of the figure there is a reference mirror, which is absolutely necessary for use with the laboratory-based LTP. The second LTP-MF optical head is mounted on the left side of the optical table and is used in conjunction with a penta-prism unit mounted on the carriage of the 1.5 meters air bearing (Fig.2 (b)). This is the penta-prism sccanning configuration and is useful for applications of precision testing of plane mirrors or near-plane mirrors. The use of a reference beam is unnecessary in this configuration. For mirrors with different curvatures we can easily switch from a laboratory-based LTP to the penta prism LTP by only changing the USB connection. In the case where the LTP-MF is used as an in-situ LTP (Fig. 2 (c)), the second LTP-MF optical head, in combination with the compact $120 \mathrm{~mm}$ mechanical slide, will be transported to the location where the in-situ test is to be performed. For angle monitoring applications (Fig. 2 (d)), the second LTP-MF optical head can also be transported to the location of test, but without the compact slide. For use as a large angle LTP (Fig. 2 (e)), the system must use the scanning optical head mode with a fixed distance to the surface under test (SUT) and with a smaller scanning distance. The laboratory-based LTP setup can be used in this case or the second optical head can be set up as another special test machine with the short and precise slide.

\section{SCANNING OPTICAL HEAD VS. SCANNING PENTA-PRISM CONSWERATIONS}

Let us look at the beam paths during a test scan. Fig. 3 (a) shows the optical path of the LTP configured with a scanning optical head. A fixed reference mirror must be used for subtracting the slide pitch error from the test data. In order to avoid overlapping the test and reference fringes, the reference mirror $R$ must be tilted by some angle in order to shift the reference fringe toward the edge of the detector. The farther the optical head moves away from the reference mirror, the farther the reference beam moves away from the optical axis as a result of the tilt angle. So the reference beam will sweep over a large region of the entrance aperture and pick up a significant amount of systematic error from all of the optical components in the beam paths, especially from the PBS and the FT lens. The returning reference beam optical paths will move from RAM to RBM then to RCM. On other hand, for a large angle surface test configuration, the sampling beam paths will only move from SP to SO then to SK. In this operation mode the sampling beam will pick up less systematic error than reference beam, because its movement across the internal glass components is smaller. The test result will include two systematic errors from the sampling beam and the reference beam. These errors are on the order of $10 \mu \mathrm{rad}(\mathrm{P}-\mathrm{V})$ and $3 \mu \mathrm{rad} \mathrm{rms}[8,9]$ respectively, which significantly compromises the accuracy of the test results. If a plane mirror is tested, and if the sampling beam is aligned in the center of the aperture so that it does not move, the reference beam tilting is still necessary. Of course, a smaller tilt angle can apply and a smaller error would result, but it is still not avoidable. This systematic error will not be acceptable for nanometer precision measurement of a plane mirror.

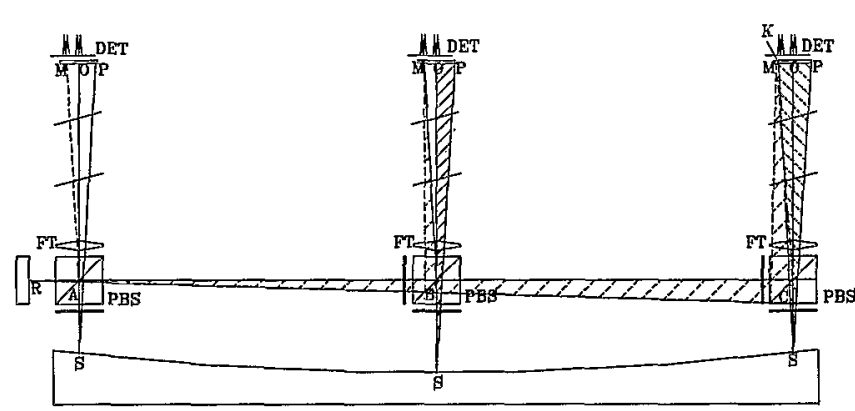

a) Optical scheme of the LTP with scanning optical head

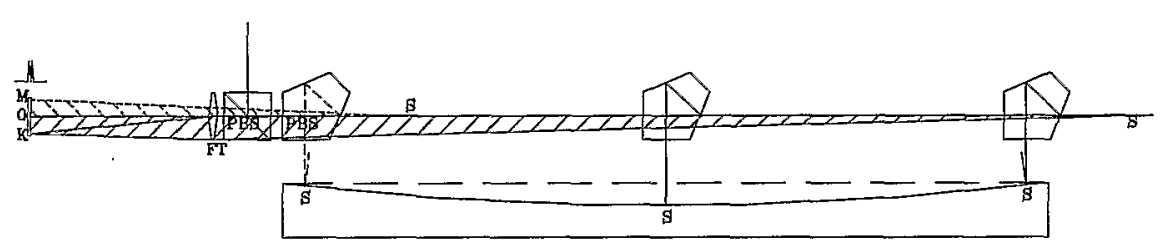

b) Optical acheme of the penta-prism LTP with fixed optical head and scanning penta-prism

Fig. 3. Optical paths of the LTPs with scanning optical head and scanning penta-prism 
Fig. 3 (b) shows the optical path of the penta-prism LTP (PPLTP) and the in-situ/portable LTP configurations. The optical head now is fixed on the table and a penta-prism is scanned from left to right. No reference mirror correction is necessary because the penta-prism eliminates the slide pitch error automatically. In this way, there will be no reference error problem like that in the scanning optical head. However, the optical paths of the sampling beam will be different from the scanning optical head (Fig. 3 (a)). When the penta-prism scans to the right, the sampling beam will move away from the center of the PBS and FT, and will pick up a larger error than in the scanning optical head configuration. In the penta-prism LTP operation mode for a plane mirror test, the single test beam should be adjusted always to be on axis during the scan, so there will be no optical path movement and hence no systematic error to be picked up from any of the optics at any time.

According to this analysis, if one expects a very precise test of a plane, or nearly-plane mirror, one must use the PPLTP configuration. But for precision measurement over a larger test range, the scanning optical head configuration is recommended because it has less sampling beam movement across the PBS and FT lens. Of course, these conditions will disappear if the reference path systematic error can be reduced significantly.

\section{SYSTEMATIC ERROR ELIMINATION IN REFERENCE ARM}

In order to solve the reference systematic error problem caused by the tilted reference beam, the LTP II profilers use a dual parallel linear array detector. The reference beam is offset onto one of the linear arrays and is completely separated from the test beam. In this case, the reference beam tilt in horizontal direction is eliminated, but it is still tilted in vertical direction, which will still produce a systematic error.

The LTP-MF applies two optical heads to eliminate the reference beam systematic error problem. The second optical head is generally set for penta-prism LTP operation. However, if we use the scanning optical head mode for testing large angle optics, the idle second optical head can be used as a reference beam to measure the slide pitch error by setting a mirror on the carriage to reflect the beam from the second optical head. In this case the first LTP-MF optical head is only used for sampling the test beam. Because the first and second LTP-MF beams are incident on the CCDs of the two different optical heads, there will no longer be a fringe overlap problem. So the reference beam can be set on the CCD center precisely to eliminate the systematic error produced by the beam sweeping across the optics. An additional benefit is that without the reference beam interference on the first optical head, the sampling test range can also be considerably expanded.

Table I - Summary of LTP measurement configurations

\begin{tabular}{|c|c|c|c|c|c|}
\hline & \multicolumn{3}{|c|}{ Curve mirror test } & \multicolumn{2}{|c|}{ Plane mirror test } \\
\hline Measurement mode & $\begin{array}{l}\text { SB lateral } \\
\text { movement }\end{array}$ & $\begin{array}{l}\mathrm{RB} \text { lateral } \\
\text { movement }\end{array}$ & $\begin{array}{l}\text { LTP optics } \\
\text { dimensions }\end{array}$ & $\begin{array}{l}\text { SB lateral } \\
\text { movement }\end{array}$ & $\begin{array}{l}\mathrm{RB} \text { lateral } \\
\text { movement }\end{array}$ \\
\hline $\begin{array}{l}\text { Scanning optical head \& } \\
\text { Reference subtraction } \\
\text { LTP II, LTP V }\end{array}$ & Smaller & Larger & Smaller & $\mathrm{NO}$ & YES \\
\hline Comment & \multicolumn{3}{|c|}{$\begin{array}{l}\text { Large slope mirror test has larger } R B \text { systematic } \\
\text { error }\end{array}$} & \multicolumn{2}{|c|}{$\begin{array}{l}\text { Not good for precise plane test } \\
\text { with } R B \text { systematic error }\end{array}$} \\
\hline $\begin{array}{l}\text { Scanning penta-prism } \\
\text { No reference } \\
\text { PPLTP, LTP-MF }\end{array}$ & Larger & NO & Larger & NO & NO \\
\hline Comment & \multicolumn{3}{|c|}{ Better for smaller slope mirror test } & \multicolumn{2}{|c|}{ Best no RB subtraction } \\
\hline $\begin{array}{l}\text { Scanning optical head } \\
\text { Extra fixed optical head } \\
\text { LTP-MF }\end{array}$ & Smaller & $\mathrm{NO}$ & Smaller & NO & NO \\
\hline Comment & \multicolumn{3}{|c|}{ Better for precise test but a little complex } & \multicolumn{2}{|c|}{ Better but with RB subtraction } \\
\hline
\end{tabular}

$\mathrm{SB}=$ sampling beam $\quad \mathrm{RB}=$ reference beam 
If the tilted reference beam of the first LTP-MF head is also recorded during a dual-head scan, we can obtain the real systematic error in the reference data by subtracting the second LTP-MF optical head data from reference data. One should be aware that this systematic error is not reproducible in general and varies according to the particular tilt angle of the reference beam and the scan position set up.

\section{LTP ACCURACY}

The LTP is capable of very precise measurements with high repeatability. It can reach sub-micro-radian absolute test, accuracy on plane mirrors with careful alignment and in a good temperature controlled environment. However, due to the quality of internal optical components, FT lens aberration and alignment, accuracy in the large angular test range is limited to the level of a few micro-radians if no additional systematic error correction procedure adopts. The large angular test accuracy can be improved to less than one micro-radian too if with systematic error correction. In any of the three measurement modes, we should be aware that the systematic error is still included in the sample beam arm, while the systematic error in the reference beam arm is eliminated in the cases of LTP-MF and Panta-prism LTP. However, we will make efforts to overcome these problems in the phase II development of the LTP-MF by improving optics quality and using error correction method to eliminate the error in the sampling beam.

\section{SCHEME OF THE LTP-MF OPTICAL HEAD}

The scheme of the LTP-MF optical head is shown on Fig. 4. With the full names of each component indicated in the figure, the LTP-MF can be understood easily.

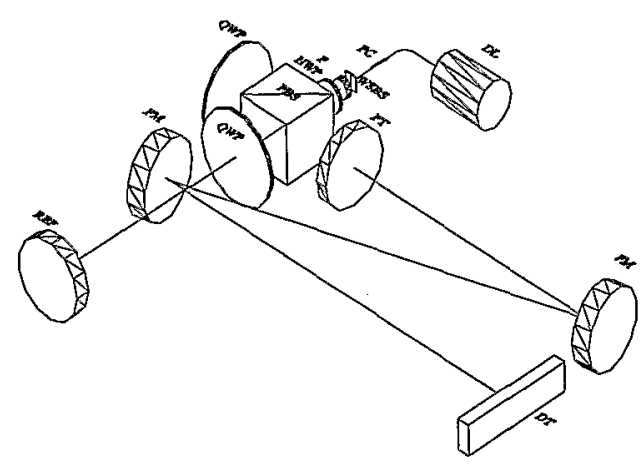

Fig. 4: Schematic of LTP-MF optical head. DL - diode laser $\mathrm{FC}$ - fiber collimator HWP - half wave plate $\quad$ QWP - quarter wave plate PBS-polarization beam splitter $\mathrm{P}$ - polarizer $\mathrm{REF}$ - reference mirror $\quad \mathrm{FM}$ - folding mirror FT - Fourier transform lens DT - detector WSBS - wavefront splitting beam splitter
We would like to emphasize following points:

1) Use of a diode laser is a low cost and compact solution.

2) It is better to use a stable monolithic beam splitter (for example, an equal optical path prism beam splitter [10] or wavefront splitting beam splitter (WSBS $[11,12]$ ) in making a high-precision LTP, due to the large frequency shift of a diode laser. We use an equal optical path WSBS by use of double cover plates for the purposes of precision and compactness.

3) The PBS should be of extremely high quality, including each surface, angles, and material uniformity to insure LTP precision.

4) In order not to produce laser beam pointing error, a fiber connection is used for isolating thermal instabilities between the diode laser and the fiber collimator.

5) Good alignment of the optical system will ensure test accuracy when the angular test range is large.

\section{USE OF THE LTP-MF FOR SMALL RADIUS OF CURVATURE MEASUREMENTS}

It is conceptually easy to enlarge the LTP measurement range by reducing the focal length, increasing the CCD size, using a high resolution CCD and by enlarging the PBS and FT aperture size. However, if sub-micro-radian measurement accuracy is required over the entire testing range then the improvement task becomes extremely difficult.

The LTP-MF uses a FT lens with a focal length of $400 \mathrm{~mm}$ and a $28 \mathrm{~mm}$ aperture, a linear detector with 2048 pixels with $14 \mu \mathrm{m}$ pitch pixels supplied by Ocean Optics, Inc., and a $20 \times 20 \mathrm{~mm}$ PBS. The LTP-MF works at a fixed distance of $200 \mathrm{~mm}$ in the scanning optical head mode. The FT lens is designed to keep aberrations below $1 \mu \mathrm{rad}$ in order to 
fulfill two conditions: point object at fixed distance of $200 \mathrm{~mm}$ with an angle of $\pm 32 \mathrm{mrad}$ (double test range for optical head scanning mode) and a variable object distance with variable angle (for tilted reference beam and sampling beam in the PPLTP mode). Due to the compact system design, the FT lens focal length has been reduced significantly from 1250 $\mathrm{mm}$ (LTP II) to $400 \mathrm{~mm}$ (LTP-MF) and due to requirement to test a small radius of curvature, the test range has been increased 3 times over that of the LTP II. In this case, the restriction of the test range is the FT aberration.

With the \pm 16 mrad angle test range, we can test an optical surface with a 1 meter radius over a 32 mm diameter without stiching. This will open the door to test telescope mirrors. For example, the primary mirror of certain telescope uses 34 mirror segments, which are all of 1.1 meters size with a $40 \mathrm{~m}$ radius of curvature, and are probably suitable for LTP-MF measurement.

\section{NEW COMPACT SLIDE FOR IN-SITU LTP}

The compact slide described previously [7] has been modified with the following improvements. A wider recirculating ball bearing slide BSGS14W supplied by DelTron Precision, Inc., is used for scanning the penta-prism. This slide is much more precise than the previous one. The compact slide specification is listed in Table II. By selecting the best one from several ball bearing slides, it is possible to have the errors of pitch, yaw and roll be less than 60 arc seconds without correction adjustments. As a matter of design, according to the penta-prism characteristics, the slide pitch will not affect the beam propagation angle, while the roll and yaw errors will produce beam spot movements of $0.24 \mathrm{~mm}$ in the vertical direction, which is not a problem for measurements with a larger Airy disk diffraction spot.

\section{STABHLITY MEASUREMENT OF THE LTP-MF}

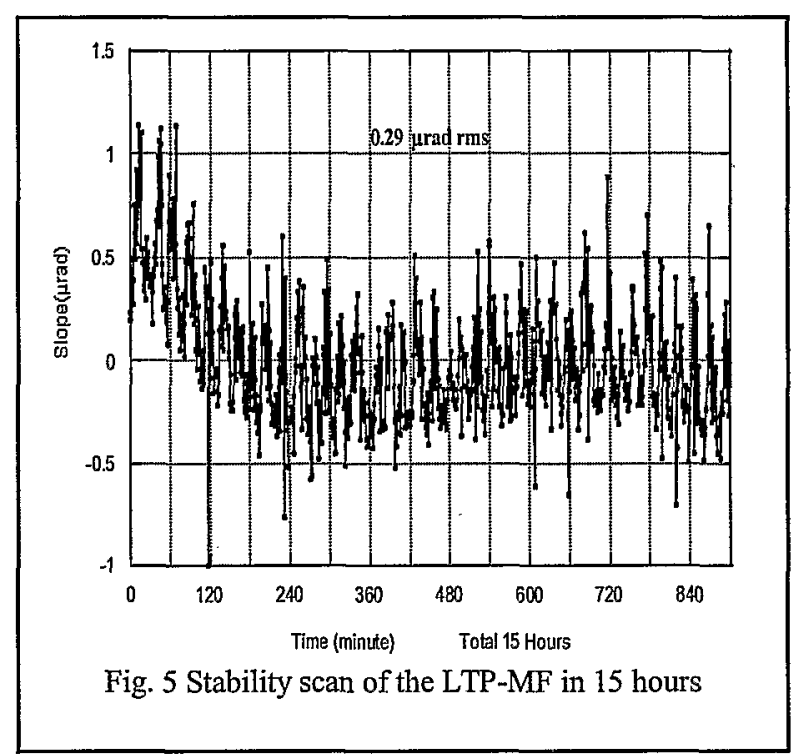

Fig. 5 is the stability scan test result of the LTP-MF of BNL. $0.29 \mu \mathrm{rad}$ rms stability in 15 hours shows that the LTP-MF is very stable. The experiment was done with LTP setting on a height adjustment jack and with a penta-prism to reflect beam to mirror under test standing on its side. The LTP-MF was in a plastic tent during the test.

\section{ACKNOWLEDGMENTS}

The authors would like to thank Ocean Optics, Inc., for providing the LTP software and the air bearing stage, and Xu Chaoyin, Liu Bin, Kan Ya and other members of NSRL for their help. This manuscript has been authored by Brookhaven Science Associates, LLC under Contract No. DE-AC0298CH10886 with the U.S. Department of Energy. The United States Government retains, and the publisher, by accepting the article for publication, acknowledges, a world-wide license to publish or reproduce the published form of this manuscript, or allow others to do so, for the United States

Government purposes. This research was sponsored in part by the U. S. Department of Energy and the National Synchrotron Radiation Laboratory, University of Science and Technology of China. 


\section{REFERENCES}

1. Peter Takacs, Shinan Qian and Jeffrey Colbert, Design of a long trace surface profiler, SPIE Vol. 749, 59-64, Aug. 1987

2. Peter Takacs, Shinan Qian, Surface profiling interferometer, US patent No.U4884697, Dec.5, 1989

3. Shinan Qian, W. Jark, P. Takacs, The penta-prism LTP: A long-trace-profiler LTP with stationary optical head and moving penta-prism, Rev. Sci. Instr. 66(3), 2562-2569, March 1995

4. Shinan Qian, W. Jark, A. Gambitta, F. Mazzolini, A. Savoia, Precise measuring method for detection the in situ distortion profile of a high-heat-load mirror for Synchrotron radiation by use of a penta-prism long trace profiler, Applied Optics, Vol. 36, No. 16, 1 June 1997

5. Shinan Qian, W. Jark, A. Gambitta, F. Mazzolini, A. Savoia, Advantages of the in-situ LTP distortion profile test on high-heat-load mirror and applications, Proc. SPIE, Vol. 2856, 1996

6. Shinan Qian, P. Takacs, Precise angle displacement monitoring system based on pencil beam interferometry, SPIE Vol. 4101, 263-272, August, 2000

7. Shinan Qian, P. Takacs, Portable Long Trace Profiler: Concept and solution, Review of Scientific Instruments, Vol.72, No. 8, 3198-3204, Aug. 2001

8. P. Takacs, Shinan Qian, Accuracy limitations in Long-Trace Profilermetry, Eighth International Conference, AIP Conference Proceeding, Vol. 705, 831-834, 2004

9. P. Takacs, Shinan Qian, Sub-microradian error sources in pencil beam interferometry, SPIE Vol.5180, 377-384, August, 2003

10. Shinan Qian, P. Takacs, Equal optical path beam splitters for a pencil beam interferometer and shearing interferometer, Opt. Eng. 42(4) 929-934, April 2003

11. Shinan Qian, P. Takacs, Equal optical path beam splitters by use of amplitude-splitting and wavefront-splitting method for pencil beam interferometer, SPIE Vol.5193, 79-88, August, 2003

12. Shinan Qian, P. Takacs, Wave front-splitting phase shift beam splitter for pencil beam interferometer, Review of Scientific Instruments, Vol.74, No. 11, 4881-4884, Nov. 2003 